\section{Fatores prognósticos}

O desenvolvimento de DVD em decorrência de um episódio de TEP é, há décadas, reconhecido como um fator de mau prognóstico. A presença de instabilidade hemodinâmica e choque, como consequência da falência ventricular direita, eleva as taxas de mortalidade dos pacientes, na fase aguda, a valores acima de 60\%.(1)

Existe certo consenso, apesar da ausência de estudos prospectivos definitivos, sobre a necessidade de se intervir de forma mais agressiva nesses quadros, com o uso de trombolíticos. Entretanto, o reconhecimento e a necessidade de intervenção na DVD sem instabilidade hemodinâmica ainda são motivo de discussão.

Em função da área do leito vascular ocluída, seja em decorrência dos êmbolos, seja em decorrência do fenômeno de vasoconstrição associado à ativação plaquetária (entre outras vias), há um aumento proporcional da resistência vascular pulmonar e, consequentemente, da pós-carga do VD. Essa sobrecarga, frente a um ventrículo não adaptado, leva ao aumento da tensão na parede do VD, assim como no volume e na pressão de enchimento diastólico, ocasionando um desvio do septo interventricular e o potencial aparecimento de fenômenos isquêmicos. Esse desvio, juntamente com a diminuição da função sistólica do VD, leva à diminuição da complacência e das pressões de enchimento do VE, respectivamente, com uma consequente diminuição do débito cardíaco, aumentando o risco de isquemia cardíaca e também do desenvolvimento de hipotensão ou choque. A maior ou menor magnitude dessa cascata de eventos está diretamente relacionada à maior ou menor mortalidade associada à TEP aguda. ${ }^{(2)}$

Entretanto, é difícil estabelecer a partir de que ponto, ou de que magnitude, a mortalidade relacionada à DVD torna-se significativa. Em outras palavras, a definição de critérios diagnósticos de DVD é extremamente importante, pois devem refletir a exata magnitude dos eventos fisiopatológicos relacionados e a mortalidade relacionadas a eles.

Essa foi sem dúvida uma das grandes dificuldades relacionadas aos estudos que avaliaram o impacto de terapias trombolíticas em pacientes com TEP aguda. 0 critério inicial, relacionado apenas à presença de instabilidade hemodinâmica, é insuficiente, uma vez que cerca de $80 \%$ dos pacientes com TEP aguda apresentam pressões arteriais sistêmicas normais, e ao menos um terço desses pacientes apresenta DVD. ${ }^{(2-5)}$ Mais ainda, enquanto a mortalidade de pacientes sem DVD, nos diferentes estudos realizados com pacientes estáveis do ponto de vista hemodinâmico, é de até 1\%, a presença de DVD eleva essa taxa a até $14 \%$, o que é bastante significativo, considerando a ausência de sinais clínicos que diferenciem essas duas situações. ${ }^{(1)}$

Alguns sinais clínicos parecem estar associados a um pior prognóstico, tais como síncope, sexo feminino, idade avançada e presença de hipoxemia. Existem escores prognósticos que auxiliam na predição do desfecho do paciente, mas que não foram utilizados a fim de orientar a terapêutica do paciente.

Várias abordagens podem ser utilizadas para o reconhecimento de DVD associada, embora a tendência atual seja a de se utilizar a combinação de dois ou mais marcadores.(D)

\section{Eletrocardiograma}

Em uma análise recente, a presença de ao menos uma alteração eletrocardiográfica compatível com sobrecarga ou isquemia relacionada ao evento embólico, como a presença de complexos de baixa voltagem, bloqueio completo de ramo direito, arritmia atrial, alterações do segmento ST nas derivações ventriculares esquerdas ou ainda alterações compatíveis com pseudoinfarto nas derivações DIll e aVF, foi associada a um maior risco de mortalidade intra-hospitalar. ${ }^{(6)}$ Embora pouco específicas, essas alterações devem servir de aviso para a necessidade de se investigar mais ativamente a função ventricular direita.(C)

\section{Ecocardiograma}

0 ecocardiograma é, sem dúvida, a ferramenta mais utilizada na avaliação da função ventricular direita. Porém, tão grande quanto a sua utilização, é a falta de padronização nos critérios diagnósticos. Os diferentes estudos que utilizaram o ecocardiograma usaram desde critérios qualitativos, como a presença de hipocinesia do VD, como quantitativos, como o aumento da relação entre o volume diastólico final do VD sobre o do $\operatorname{VE}(>1)$ ou o aumento isolado do volume diastólico final do VD (>30 mm), ou ainda sinais de hipertensão pulmonar com o aumento da pressão sistólica da artéria pulmonar a valores acima de $30 \mathrm{mmHg}$ ou da pressão média da artéria pulmonar a valores acima de 20 mmHg. ${ }^{(3,4,7,8)} 0$ achado de qualquer um desses sinais foi relacionado a uma mortalidade associada ao evento agudo da embolia, até duas 
vezes maior. Mais recentemente, confirmou-se o papel da avaliação ecocardiográfica através da análise dos pacientes incluídos no International Cooperative Pulmonary Embolism Registry.(C) ${ }^{(7)}$

$\mathrm{Na}$ subpopulação estudada, a presença de hipocinesia do VD demonstrou ser um fator independente de mortalidade $(O R=1,94$; 1C95\%: 1,23-3,06)..$^{(9)}$ De qualquer forma, existe ainda a necessidade de padronização quanto aos critérios utilizados e seus respectivos valores de corte para a caracterização de DVD através da avaliação ecocardiográfica.

\section{Angio-TC de tórax}

À medida que a angio-TC foi se tornando o exame de escolha na avaliação diagnóstica da embolia pulmonar, cresceu o interesse em se utilizar o mesmo exame para a avaliação do VD, principalmente no que se refere ao aumento da cavidade ventricular. ${ }^{(10)} \mathrm{Um}$ estudo retrospectivo demonstrou que o aumento do VD, avaliado pela angio-TC de tórax, através da relação entre as dimensões do VD e VE (anormal quando $>0,9)$, é um fator independente de mortalidade $(\mathrm{OR}=5,17 ; 1 \mathrm{C} 95 \%$ : 1,63-16,35), confirmando assim o potencial uso dessa ferramenta na estratificação de risco dos pacientes com embolia pulmonar aguda.(C) $)^{(11)}$

\section{Marcadores bioquímicos}

De forma análoga ao que aconteceu com a avaliação da disfunção ventricular esquerda, o aparecimento de marcadores bioquímicos, como os peptídeos natriuréticos e a troponina, demonstrou ser de extrema utilidade na avaliação da DVD, tanto aguda quanto crônica.

A elevação dos níveis de troponina, como marcador de lesão de cardiomiócitos, não só se correlaciona com a extensão de DVD (medida através do ecocardiograma), como também é um marcador independente de mortalidade.(B) (12) Já a elevação dos níveis de peptídeos natriuréticos (tanto o BNP quanto o NT-proBNP), liberados pelos cardiomiócitos em decorrência do aumento da tensão na parede ventricular, apresenta VPNs ainda maiores que os da troponina. Apesar de esse achado ser bastante significativo, a elevação dos níveis de troponina ou dos peptídeos natriuréticos não possui VPP suficiente para identificar os pacientes hemodinamicamente estáveis que possam se beneficiar do tratamento trombolítico, por exemplo.(B) ${ }^{(13)}$ A combinação desses dois marcadores parece melhorar seu potencial de estratificação de risco, embora a análise de custo-efetividade ainda seja necessária. Um estudo publicado em 2005, com pacientes com embolia pulmonar e sem instabilidade hemodinâmica, mostrou que níveis baixos de NT-proBNP caracterizavam um grupo de baixo risco (relacionado à embolia); já pacientes com NT-proBNP aumentado, mas com troponina normal, caracterizavam um grupo de risco intermediário, enquanto pacientes com o aumento de ambos os marcadores apresentavam alto risco, com mortalidade associada à embolia

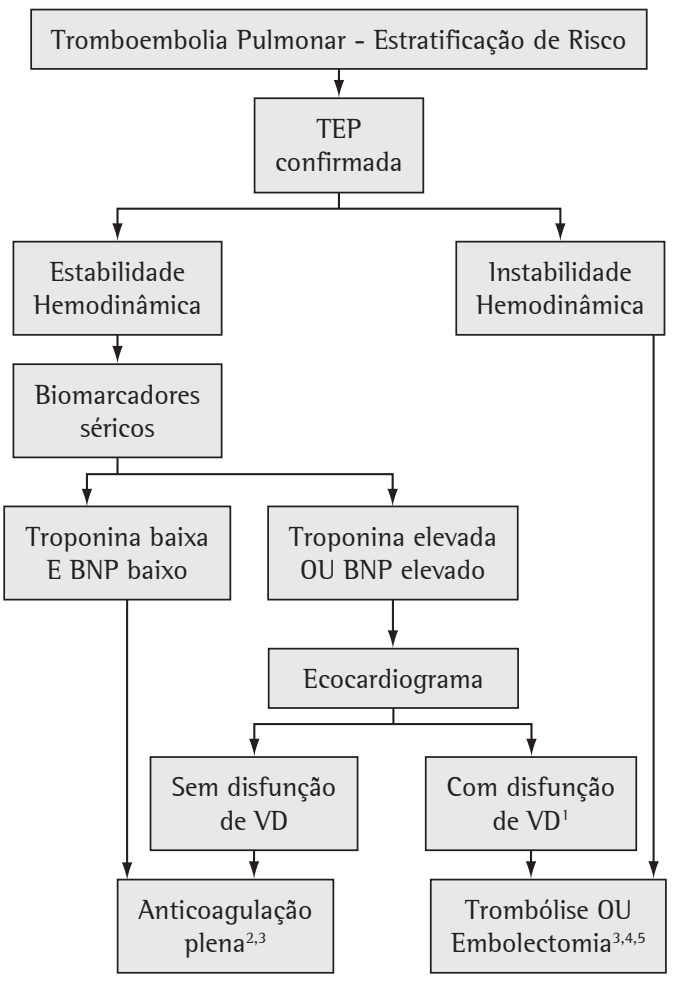

'Disfunção de ventriculo direito definida como dilatação de VD, hipocinesia de VD, presença de hipertensão pulmonar (PSAP > $30 \mathrm{mmHg}$ ). Na decisão terapêutica, considerar a limitação deste método pelo grau de subjetividade de alguns destes critérios.

${ }^{2}$ Em pacientes estáveis, dar preferência as HBPM para a anticoagulação plena.

${ }^{3} \mathrm{~A}$ decisão terapêutica final depende de contraindicação às terapias e da disponibilidade local.

${ }^{4}$ Alguns fatores clinicos, tais como presença de síncope, hipoxemia e idade avançada podem contribuir para a decisão de tratamento mais agressivo

IIniciar HNF i.v. em infusão contínua (sem bolus), conforme TTPa seriado após uso do trombolítico

Figura 3 - Algoritmo de estratificação de risco de pacientes com TEP aguda. BNP: brain natriuretic peptide; e PSAP: pressão sistólica da artéria pulmonar. 
de até 33\%.(B) ${ }^{(14)}$ Em duas meta-análises, foi confirmada a importância da troponina e de BNP na estratificação de risco de TEP. ${ }^{(15,16)} \mathrm{Em}$ um recente estudo prospectivo, no qual foram dosados oito biomarcadores, demonstrou-se que somente a troponina e BNP tinham a capacidade para predizer a mortalidade na TEP. ${ }^{(17)}$ Mais uma vez, porém, é necessário ressaltar as limitações relacionadas à especificidade desses marcadores em relação à embolia pulmonar.

\section{Caracterização combinada}

Deve-se considerar que a ausência de critérios homogêneos, a depender do método diagnóstico utilizado, ou de estudos prospectivos que os validem, não permite ainda a recomendação de um algoritmo para a avaliação da função ventricular direita frente ao quadro de embolia pulmonar aguda, mais especificamente em pacientes hemodinamicamente estáveis. Entretanto, o resultado de estudos recentes, combinando a análise de marcadores bioquímicos e exames de imagem, como o ecocardiograma, levou à proposição de um algoritmo a ser utilizado nos próximos estudos prospectivos, a fim de ser validado quanto a sua capacidade diagnóstica e de estratificação de risco (Figura 3).(C) $)^{(13,18,19)}$ Esse algoritmo leva em consideração a sensibilidade dos marcadores bioquímicos e a especificidade do ecocardiograma, tentando, dessa forma, alcançar o maior compromisso entre o VPP e o VPN para a identificação de pacientes com DVD e, até mesmo, para a proposição de terapia trombolítica. A utilização desse algoritmo, porém, ainda depende de sua validação, principalmente no que se refere à utilização de trombolíticos, podendo ser utilizado tão somente para a caracterização de DVD associada à embolia pulmonar aguda.

\section{Referências}

1. Kasper W, Konstantinides S, Geibel A, Olschewski M, Heinrich F, Grosser KD, et al. Management strategies and determinants of outcome in acute major pulmonary embolism: results of a multicenter registry. J Am Coll Cardiol. 1997;30(5):1165-71.

2. Lualdi JC, Goldhaber SZ. Right ventricular dysfunction after acute pulmonary embolism: pathophysiologic factors, detection, and therapeutic implications. Am Heart J. 1995;130(6):1276-82.

3. Grifoni S, Olivotto I, Cecchini P, Pieralli F, Camaiti A, Santoro G, et al. Short-term clinical outcome of patients with acute pulmonary embolism, normal blood pressure, and echocardiographic right ventricular dysfunction. Circulation. 2000;101(24):2817-22.

4. Ribeiro A, Lindmarker P, Juhlin-Dannfelt A, Johnsson $\mathrm{H}$, Jorfeldt L. Echocardiography Doppler in pulmonary embolism: right ventricular dysfunction as a predictor of mortality rate. Am Heart J. 1997;134(3):479-87.

5. Wolfe MW, Lee RT, Feldstein ML, Parker JA, Come PC, Goldhaber SZ. Prognostic significance of right ventricular hypokinesis and perfusion lung scan defects in pulmonary embolism. Am Heart J. 1994;127(5):1371-5.

6. Geibel A, Zehender M, Kasper W, Olschewski M, Klima C, Konstantinides SV. Prognostic value of the ECG on admission in patients with acute major pulmonary embolism. Eur Respir J. 2005;25(5):843-8.

7. Goldhaber SZ, Visani L, De Rosa M. Acute pulmonary embolism: clinical outcomes in the International Cooperative Pulmonary Embolism Registry (ICOPER). Lancet. 1999;353(9162):1386-9.

8. Kasper W, Konstantinides S, Geibel A, Tiede N, Krause $\mathrm{T}$, Just $\mathrm{H}$. Prognostic significance of right ventricular afterload stress detected by echocardiography in patients with clinically suspected pulmonary embolism. Heart. 1997;77(4):346-9.

9. Kucher N, Rossi E, De Rosa M, Goldhaber SZ. Prognostic role of echocardiography among patients with acute pulmonary embolism and a systolic arterial pressure of 90 mm Hg or higher. Arch Intern Med. 2005;165(15):177781.

10. British Thoracic Society Standards of Care Committee Pulmonary Embolism Guideline Development Group. British Thoracic Society guidelines for the management of suspected acute pulmonary embolism. Thorax. 2003;58(6):470-83.

11. Schoepf UJ, Kucher N, Kipfmueller F, Quiroz R, Costello $\mathrm{P}$, Goldhaber SZ. Right ventricular enlargement on chest computed tomography: a predictor of early death in acute pulmonary embolism. Circulation. 2004;110(20):3276-80.

12. Douketis JD, Leeuwenkamp O, Grobara P, Johnston M, Söhne M, Ten Wolde M, et al. The incidence and prognostic significance of elevated cardiac troponins in patients with submassive pulmonary embolism. J Thromb Haemost. 2005;3(3):508-13.

13. Kucher N, Goldhaber SZ. Cardiac biomarkers for risk stratification of patients with acute pulmonary embolism. Circulation. 2003;108(18):2191-4.

14. Kostrubiec M, Pruszczyk P, Bochowicz A, Pacho R, Szulc M, Kaczynska A, Biomarker-based risk assessment model in acute pulmonary embolism. Eur Heart J. 2005;26(20):2166-72.

15. Becattini C, Vedovati MC, Agnelli G. Prognostic value of troponins in acute pulmonary embolism: a metaanalysis. Circulation. 2007;116(4):427-33.

16. Coutance G, Le Page 0, Lo T, Hamon M. Prognostic value of brain natriuretic peptide in acute pulmonary embolism. Crit Care. 2008;12(4):R109.

17. Kline JA, Zeitouni R, Marchick MR, Hernandez-Nino J, Rose GA. Comparison of 8 biomarkers for prediction of right ventricular hypokinesis 6 months after submassive pulmonary embolism. Am Heart J. 2008;156(2):308-14.

18. Torbicki A, Perrier A, Konstantinides S, Agnelli G, Galiè $\mathrm{N}$, Pruszczyk P, et al. Guidelines on the diagnosis and management of acute pulmonary embolism: the Task Force for the Diagnosis and Management of Acute Pulmonary Embolism of the European Society of Cardiology (ESC). Eur Heart J. 2008;29(18):2276-315.

19. Kearon C, Kahn SR, Agnelli G, Goldhaber S, Raskob GE, Comerota AJ, et al. Antithrombotic therapy for venous thromboembolic disease: American College of Chest Physicians Evidence-Based Clinical Practice Guidelines (8th Edition). Chest. 2008;133(6 Suppl):454S-545S. 